Silvino, VO, Pereira, MML, Moura, RC, Batista, MCC, Rosa, BV, Moura, EH, Martins, MCC \& Santos, MAP (2020). Vitamin D and infectious diseases in the COVID-19 pandemic outburst. Research, Society and Development, 9(7): 1-21, e771974614.

\title{
Vitamina D e doenças infectocontagiosas na pandemia da COVID-19
}

Vitamin D and infectious diseases in the COVID-19 pandemic outburst

Vitamina D y enfermedades infectocontagiosas en la pandemia de COVID-19

Recebido: 17/05/2020 | Revisado: 26/05/2020 | Aceito: 28/05/2020 | Publicado: 12/06/2020

Valmir Oliveira Silvino

ORCID: https://orcid.org/0000-0002-1992-5199

Universidade Federal do Piauí, Brasil

E-mail: valmirsilvino@live.com

Mayara Maria Lima Pereira

ORCID: https://orcid.org/0000-0001-5641-7591

Universidade Federal do Piauí, Brasil

E-mail: hello_mayara@hotmail.com

Rayane Carvalho de Moura

ORCID: https://orcid.org/0000-0003-0188-9385

Universidade Federal do Piaú, Brasil

E-mail: rayane_cm@hotmail.com

Mara Cristina Carvalho Batista

ORCID: https://orcid.org/0000-0001-7743-7516

Universidade Federal do Piauí, Brasil

E-mail: maracristinacb@ hotmail.com

Bruno Viana Rosa

ORCID: https://orcid.org/0000-0003-2215-5153

Universidade Federal do Piauí, Brasil

E-mail: brunovianarosa@gmail.com

Eduardo Henrique de Moura

ORCID: https://orcid.org/0000-0001-7574-1098

Universidade Estadual do Piauí, Brasil

E-mail: dudu.uespi@gmail.com 


\section{Maria do Carmo Carvalho e Martins}

ORCID: https://orcid.org/0000-0002-9107-2485

Universidade Federal do Piaú, Brasil

E-mail: carminhamartins@ufpi.edu.br

Marcos Antonio Pereira dos Santos

ORCID: https://orcid.org/0000-0002-0755-6138

Universidade Federal do Piauí, Brasil

E-mail: marcosedfisio@gmail.com

\section{Resumo}

A pandemia da doença do novo coronavírus (COVID-19) surgiu no fim de 2019 em Wuhan, China, e rapidamente se alastrou ao redor de todo o globo. Considerando sua rápida taxa de transmissão, a COVID-19 tornou-se uma ameaça à saúde pública mundial. Portanto, ideias objetivas baseadas em evidências científicas devem ser propostas para o tratamento de pacientes infectados. A vitamina $\mathrm{D}$ é um hormônio que apresenta função importante na regulação da função do sistema imune. As principais fontes de obtenção da Vitamina D são a alimentação e do processo de síntese no organismo iniciado na pele, através de fotorreação mediada pela luz solar, em que a pré-vitamina $\mathrm{D}$ isomeriza-se em pró-vitamina $\mathrm{D}$. Posteriormente, é metabolizada no fígado em 25-hidroxivitamina D, e convertida no rim em sua forma ativa, a 1,25-dihidroxivitamina D. Por conta do seu papel chave na melhoria da resposta imune, a Vitamina D pode afetar positivamente o tratamento de diversas doenças infectocontagiosas, tais como a influenza e H1N1. Esta revisão tem como objetivo informar sobre o uso de Vitamina D como possível aliado no tratamento da COVID-19. Os estudos incluídos nesta revisão apontam que a vitamina D pode exercer funções imunomoduladoras e anti-inflamatórias, que beneficiam significativamente o tratamento de infecções virais. Recomenda-se que ensaios clínicos randomizados e estudos populacionais amplos sejam conduzidos para melohr elucidar o papel efetivo da Vitamina D no tratamento de pacientes com COVID-19.

Palavras-chave: SARS; Coronavírus; Tratamento; H1N1; Influenza.

\section{Abstract}

The new coronavirus pandemic disease (COVID-19) pandemic was first reported in Wuhan, China, in late 2019, and rapidly spread around the world. Considering its fast transmission rate, the new 
COVID-19 has become a threat to the public health worldwide. Thus, objective ideas based on scientific evidences must be proposed for the treatment of patients infected. Vitamin D is a hormone with important role in the regulation of the immune system functioning. The main sources of Vitamin $\mathrm{D}$ are food intake and synthesis process in the organism which starts in the skin through photoreaction with sun exposure. Then, the pre-Vitamin D is isomerized into pro-Vitamin D. Afterwards, it is metabolized in the liver into 25-hydroxivitamin $\mathrm{D}$, and then converted into in the kidney into its active form, the 1.25-dihydroxivitamin D. Due to its pivotal role in the improvement of the immune response, Vitamin D can positively affect the treatment of several infecto-contagious diseases, such as influenza and H1N1. This concise review aims to inform about the use of Vitamin D as a possible ally in the treatment of COVID-19. The studies included in this review point out that Vitamin D can perform immunomodulatory and anti-inflammatory functions, which would significantly improve the treatment of viral infections. Further clinical trials and large populational studies should be carried out in order to better elucidate the effective role of Vitamin D in the treatment of patients with COVID-19.

Keywords: SARS; Coronavirus; Treatment; H1N1; Influenza.

\section{Resumen}

La nueva pandemia de la enfermedad coronavirus (COVID-19) surgió a fines de 2019 en Wuhan, China, y se extendió rápidamente por todo el mundo. Teniendo en cuenta su velocidad de transmisión rápida, COVID-19 se ha convertido en una amenaza para la salud pública en todo el mundo. Por lo tanto, se deben proponer ideas objetivas basadas en evidencia científica para el tratamiento de pacientes infectados. La vitamina $\mathrm{D}$ es una hormona que juega un papel importante en la regulación de la función del sistema inmune. Las principales fuentes de obtención de vitamina D son los alimentos y el proceso de síntesis en el cuerpo iniciado en la piel, a través de la fotorreacción mediada por la luz solar, en la que la pre-vitamina D se isomeriza en pro-vitamina D. Posteriormente, se metaboliza en el hígado a 25-hidroxivitamina $\mathrm{D}$, y convertida al riñón en su forma activa, 1,25-dihidroxivitamina D. Debido a su papel clave en la mejora de la respuesta inmune, la vitamina D puede afectar positivamente el tratamiento de diversas enfermedades infecciosas, tales como influenza y H1N1. Esta revisión tiene como objetivo informar sobre el uso de vitamina $\mathrm{D}$ como un posible aliado en el tratamiento de COVID-19. Los estudios incluidos en esta revisión indican que la vitamina D puede tener funciones inmunomoduladoras y antiinflamatorias, lo que beneficia significativamente el tratamiento de las infecciones virales. Se recomienda que se realicen ensayos clínicos aleatorios y grandes estudios de población para ayudar a dilucidar el papel efectivo de la vitamina D en el tratamiento de pacientes con COVID-19.

Palabras clave: SARS; Coronavirus; Tratamiento; H1N1; Influenza. 


\section{Introdução}

A doença do coronavírus 2019 (COVID-19) é uma infecção viral provocada pelo Coronavírus 2 da Síndrome Respiratória Aguda Grave (SARS-CoV-2). A doença surgiu em Wuhan, China, em dezembro de 2019, rapidamente espalhando-se em todas as regiões ao redor do mundo (Kimball et al., 2020). Pacientes infectados com SARS-CoV-2 podem apresentar diversos sintomas, tais como fadiga muscular, falta de ar, tosse seca e febre (Yan, Faraji, Prajapati, Boone, \& DeConde, 2020). Há evidências de que o sistema imunológico possui um papel fundamental no combate à Síndrome Respiratória Aguda Grave - SARS (Ou et al., 2020). De fato, o sistema imune inato é capaz de reconhecer as estruturas moleculares produzidas pela infecção por SARS-CoV-2 (Rokni, Ghasemi, \& Tavakoli, 2020). Portanto, o fortalecimento do sistema imunológico representa um importante papel no combate à COVID-19.

Diante do cenário mundial resultante da ameaça da infecção pelo novo coronavírus, é fundamental usar todas as estratégias para evitar o contágio e manter as pessoas saudáveis. Para isso, importa primeiramente seguir todas as recomendações da Organização Mundial de Saúde (OMS) relativas à prevenção da sua transmissão, incluindo medidas como: distanciamento social, cumprimento da etiqueta respiratória e reforço das medidas de higiene. Além disso, ter uma alimentação saudável e segura é essencial para a manutenção do bem estar geral da população, bem como para assegurar um adequado estado nutricional, considerado fundamental para a recuperação em caso de infecção (Oliveira et al., 2020).

A alimentação é essencial na prevenção e controle das doenças crônicas, bem como de doenças infectocontagiosas, pois é necessária para o bom funcionamento do sistema imune. Isso se deve ao fato de esse sistema necessitar de energia e de um vasto conjunto de nutrientes para funcionar adequadamente, tais como proteína, ácidos graxos ômega 3, cobre, folato, ferro, selênio, zinco, e vitaminas A, B6, B12, C e D (Katona \& Katona-Apte, 2008).

Dentre os nutrientes associados ao bom funcionamento do sistema imune, a vitamina D tem recebido grande destaque por conta de seus efeitos positivos sobre a imunidade e no combate de infecções virais do trato respiratório superior (Grant et al., 2020a). Diferentes tipos de informação sobre possíveis benefícios de suplementos de vitamina D para a prevenção da covid-19 têm sido amplamente divulgadas em redes sociais e veículos de comunicação de massa, em sua maioria por pessoas leigas e sem nenhum embasamento científico. Diante do exposto, esta revisão tem como objetivo informar sobre o uso de Vitamina D como possível aliado no tratamento da COVID-19. 


\section{Metodologia}

O estudo é uma revisão narrativa baseada em um levantamento bibliográfico de artigos nas bases de dados eletrônicas PubMed, Scientific Electronic Library Online (Scielo), Scopus, ScienceDirect e Google Scholar, selecionados em maio de 2020. As palavras-chave utilizadas para a pesquisa foram coronavírus, COVID-19, SARS-CoV-2, vitamina D, resposta imune e síndromes gripais, tanto na língua portuguesa quanto na língua inglesa. Os descritores foram usados sozinhos ou em combinação usando os operadores booleanos AND e OR.

Foram incluídos estudos que apresentaram informações relevantes acerca da possibilidade do uso de Vitamina D no tratamento de pacientes infectados com COVID-19. Foram incluídos artigos em língua portuguesa e inglesa. A seleção dos artigos foi baseada na originalidade e relevância, preferindo artigos mais recentes. Dissertações, teses, artigos não disponíveis na íntegra e / ou duplicados em diferentes bases de dados foram excluídos.

Em seguida, os artigos incluídos foram analisados por meio da leitura dos títulos, seguidos de seus resumos e, posteriormente, do texto completo. Em todos os estágios, os critérios de exclusão foram aplicados em consenso com os revisores.

\section{Resultados e Discussão}

\subsection{Mecanismo de produção e ação da Vitamina $D$}

A vitamina D é uma vitamina lipossolúvel essencial, que pode ser obtida na alimentação e ser sintetizada pelo organismo através de fotorreação mediada pela luz solar, em que a pré-vitamina D isomeriza-se em pró-vitamina D. Posteriormente, é metabolizada no fígado em 25-hidroxivitamina D e convertida no rim em sua forma ativa, a 1,25dihidroxivitamina D (Holick, 1995). A síntese de vitamina D na pele é a fonte mais importante de vitamina $\mathrm{D}$ e depende da intensidade da irradiação ultravioleta além de determinantes tais como a estação e latitude (Christakos, Dhawan, Verstuyf, Verlinden, \& Carmeliet, 2016).

As duas formas primárias de vitamina D (D2 e D3) são obtidas em fontes alimentares de origem vegetal, enquanto a forma D3 é obtida a partir fontes animais e a partir de processo de síntese por exposição de substrato precursor, o 7-desidrocolesterol, aos raios ultravioleta para formar a vitamina D3 (Chiang, Ismaeel, Griffis, \& Weems, 2016). 
Essa vitamina D pode ser absorvida dos alimentos como colecalciferol (vitamina D3) ou ergocalciferol (vitamina D2). A diferença básica é na sua estrutura, sendo a vitamina D3 mais facilmente absorvida no intestino do que a vitamina D2 (Borella, Nesher, Israeli, \& Shoenfeld, 2014), e sua excreção ocorre sobretudo pela vesícula biliar (Sociedade Brasileira de Pediatria, 2014).

Para exercer suas ações fisiológicas a vitamina D produzida na pele ou obtida na alimentação é convertida na forma ativa por meio de reações de hidroxilação, sendo que a primeira hidroxilação ocorre no fígado e consiste na conversão do colecalciferol 25hidroxivitamina D (25-OH-D) por ação da enzima D3-25-hidroxilase (Prosser \& Jones, 2004). Posteriormente, a 25-OH-D é metabolizada em $1,25(\mathrm{OH})_{2} \mathrm{D}$ por meio da enzima 25(OH)1a-hidroxilase em células renais e também em células de outros tecidos e órgãos, células epiteliais, células do sistema imunológico e das glândulas paratireoides (Bikle, Malmstroem, \& Schwartz, 2017).

A produção renal de 1,25 $(\mathrm{OH})_{2} \mathrm{D}$ é estimulada pelo hormônio das paratireoides e é inibida por ação do cálcio, fosfato e fator de crescimento fibroblástico 23 (FGF-23). Já a produção extrarrenal de 1,25 $(\mathrm{OH})_{2} \mathrm{D}$, como ocorre em queratinócitos e os macrófagos está sob controle diferente, sendo estimulada primariamente por citocinas, a exemplo do fator de necrose tumoral alfa $(\mathrm{TNF} \alpha)$ e interferon $\gamma(\mathrm{IFN}-\gamma)$. A 1,25 (OH) ${ }_{2} \mathrm{D}$, ou calcitriol, reduz os níveis de 1,25 $(\mathrm{OH})$ 2D nas células e estimula seu catabolismo através da indução do CYP24A1A, a 24-hidroxilase. A 25OHD e 1,25 (OH) 2 D são hidroxiladas na posição 24 por essa enzima para formar 24,25 $(\mathrm{OH})_{2} \mathrm{D}$ e 1,24,25 $(\mathrm{OH})_{3} \mathrm{D}$, respectivamente (Bikle, Patzek, \& Wang, 2018),

A 24-hidroxilação é geralmente o primeiro passo no catabolismo desses metabólitos ativos para o produto final do ácido calcitroico. As diversas espécies diferem em sua razão de atividade da 23-hidroxilase/24-hidroxilase na enzima CYP24A1, mas a atividade da 24hidroxilase predomina em humanos. A CYP24A1 é induzida por 1,25 $(\mathrm{OH})_{2} \mathrm{D}$, mecanismo esse muito importante, pois atua de forma a diminuir o risco de toxicidade da vitamina $\mathrm{D}$ (Sakaki et al., 2000).

Os metabólitos da vitamina $\mathrm{D}$ são transportados no sangue ligados à proteína transportadora de vitamina $\mathrm{D}$ (DBP) e à albumina, enquanto uma pequena fração é transportada de forma livre. O fígado produz DBP e albumina, que diminui na doença 
hepática, e essas proteínas podem ser perdidas nas enteropatias perdedoras de proteínas ou na síndrome nefrótica (Bikle et al., 2017; Schwartz, Gallagher, \& Jorde, 2018).

$\mathrm{O}$ receptor para $1,25(\mathrm{OH})_{2} \mathrm{D}(\mathrm{VDR})$ é um fator de transcrição que regula a expressão de genes que medeiam a sua atividade biológica. O VDR é amplamente distribuído não somente nos tecidos-alvo clássicos da vitamina $\mathrm{D}$. Após a ligação ao $1,25(\mathrm{OH}){ }_{2} \mathrm{D}$, o VDR sofre heterodimerização com outros receptores de hormônios nucleares, em particular a família de receptores $X$ retinóides. Esse complexo então se liga a sequências específicas do DNA chamadas VDRE (elementos de resposta à vitamina D) (Carlberg, 2017; Whitfield, 1996).

Existem milhares de VDREs em centenas de genes, e o perfil de VDREs ativos (e genes regulados) varia de célula para célula. Uma variedade de proteínas adicionais chamadas correguladores sofre complexação com o VDR para ativar (coativadores) ou inibir (correpressores) a atividade transcricional do VDR. Os coativadores podem formar uma ponte da ligação do VDR ao VDRE com proteínas como a RNA polimerase II no local de início da transcrição ou ajudar a desvendar a cromatina no local do gene por meio do recrutamento de histona acetil transferases (HAT) e desmetilases (DME), permitindo que a transcrição continue (Bikle et al., 2017).

O complexo de proteína que interage com o receptor de vitamina D (DRIP) é um tipo de complexo coativador, no qual a família coativadora de receptores esteroides (SRC 1-3) é exemplo do último tipo de coativador. Além dos coativadores, existem vários correpressores. Os correpressores geralmente trabalham recrutando histona desacetilases (HDAC) ou metil transferases (MT) para o gene que inverte as ações do HAT e DME, levando a uma redução no acesso ao gene pelo mecanismo de transcrição (McKenna et al., 2009; Oda et al., 2003).

A $1,25(\mathrm{OH})_{2} \mathrm{D}$ exerce diversas funções por meio de regulação da expressão gênica, além de ações não genômicas, incluindo a capacidade de estimular o transporte de cálcio através da membrana plasmática, com destaque para a sua participação com a homeostase do mesmo. Além disso, entre as ações da $1,25(\mathrm{OH})_{2} \mathrm{D}$ também estão a regulação da proliferação e diferenciação celular, a regulação da secreção hormonal e a regulação da função imune, levando ao desenvolvimento de um número de análogos na esperança de tratamento de desordens hiperproliferativas (Haussler, Jurutka, Mizwicki, \& Norman, 2011). 
A vitamina D é um importante hormônio e apresenta função primária na regulação homeostática de cálcio, além de outras funções secundárias, incluindo a participação na regulação da função imunológica e da síntese proteica (Trochoutsou, Kloukina, Samitas, \& Xanthou, 2015). Essa vitamina vem recebendo grande atenção atualmente em razão da demonstração de que sua deficiência apresenta relação com uma maior vulnerabilidade a infecções em consequência de prejuízos na imunidade inata e na resposta imune celular específica do antígeno (Wintergerst, Maggini, \& Hornig, 2007). Além disso, há evidências de que a 1,25-di-hidroxivitamina D3 [1,25 $(\mathrm{OH}) 2 \mathrm{D} 3]$, forma ativa de vitamina $\mathrm{D}$, gera uma série de respostas biológicas associadas ao combate de alguns tipos de cânceres, doenças autoimunes, e está associada com uma resposta positiva em doenças infectocontagiosas. Neste cenário atual em que a COVID-19 vem atingindo grande número de pessoas em diferentes países do mundo, a busca por maior entendimento de estratégias de combate a essa doença faz-se necessária (Haussler et al., 2012).

\subsection{Vitamina D e resposta imune}

O sistema imune é o mecanismo primário de defesa do organismo contra patógenos e perturbações no organismo. Falhas no sistema imune nas funções defensivas podem causar diversos distúrbios, dentre eles o desenvolvimento de doenças (Scully, Georgakopoulou, \& Hassona, 2017). A capacidade do corpo humano em se proteger de agentes invasores infecciosos pode ser classificada em imunidade inata ou adquirida (Castranova, Asgharian, Sayre, Virginia, \& Carolina, 2014).

O papel da vitamina D no sistema imune tem sido explorado em razão do fato de que além de regular o cálcio e a saúde óssea, foi demonstrado que ela atua em diversas vias do sistema imunológico, que leva à diminuição da produção de citocinas pró-inflamatórias e aumenta a expressão de citocinas anti-inflamatórias (Gombart, Pierre, \& Maggini, 2020). O receptor de vitamina $\mathrm{D}$ está presente em vários tipos de células do sistema imune, como células $\mathrm{T}$, linfócitos $\mathrm{B}$, células dendríticas e células apresentadoras de antígeno. Ademais, tanto de forma sistêmica quanto gerada localmente, a vitamina $\mathrm{D}$, em sua forma ativa, pode atuar por meio de sinalização endócrina, parácrina e autócrina, interagindo com receptores de vitamina D expressos pelas células do sistema imune (Haroon \& FitzGerald, 2011; Wu, Lewis, Pae, \& Meydani, 2019).

Em infecções agudas do trato respiratório a suplementação com vitamina D foi eficiente para diminuir a frequência de infecções, apesar da dose e do uso para o tratamento 
dessas infecções ainda ser questionado (Rondanelli et al., 2018). Além disto, em estudo com gestantes suplementação de vitamina $\mathrm{D}$ em gestantes foi demonstrado aumento do peso ao nascer e modulação da resposta imune, que pode favorecer a proteção contra patógenos (Hornsby et al., 2018).

A vitamina D atua no sistema imune inato aumentando tanto a capacidade quimiotática quanto fagocítica das suas células (Lin, 2016). O principal mecanismo pelo qual a 1,25-di-hidroxivitamina $\mathrm{D}$ interage com a resposta imune inata é ativar as catelicidinas, que fazem parte de uma família de peptídeos antimicrobianos em macrófagos e leucócitos polimorfonucleares. E, baixos níveis circulantes de vitamina $\mathrm{D}$ em algumas populações têm sido relacionados à diminuição da indução de catelicidina e a um risco aumentado de infecção tuberculosa (Czaja \& Montano-Loza, 2018).

A vitamina D não apenas modula os monócitos como também é importante para outras células apresentadoras de antígenos, especialmente as células dendríticas, atuando nas células que ligam os dois braços da imunidade, inata e adaptativa (Lin, 2016; Wu et al., 2019). A vitamina $D$ exerce efeitos diretos nas células $T$ e $B$ e também modifica suas respostas à ativação, desempenhando importante papel nas respostas imunes adaptativas (Guillot, Semerano, Saidenberg-Kermanac'h, Falgarone, \& Boissier, 2010).

Nas células T essa vitamina inibe a resposta relacionada a TH1 e estimula a resposta TH2. Desta forma, aumenta a produção de IL-5 e IL-10, bem como indiretamente, diminui a produção de IFN- $\gamma$ (Guillot et al., 2010). Além disto, a suplementação de vitamina mostrou aumentar a proporção de linfócitos $\mathrm{T}$ regulatórios (TReg cell) circulantes. Também foi observado aumento da capacidade supressora de TReg cell (\% de proliferação de células T efetoras na presença de TReg cell autólogos) e isso pode ter papel fundamental no tratamento de doenças autoimunes (Fisher et al., 2019).

Ainda em relação ao sistema imune adaptativo, no que diz respeito a células $\mathrm{B}$, a vitamina $\mathrm{D}$ diminui a sua diferenciação para células plasmáticas, diminui a produção de imunoglobulina e inibe a proliferação de células B ativadas, mas não a apoptose das mesmas (Chen et al., 2007). Portanto, a vitamina D parece atuar na imunidade de diversas formas.

\subsection{Vitamina $D$ e síndromes gripais}

As síndromes gripais (SG), responsáveis pelas doenças respiratórias comuns na maioria da população mundial, podem ter diferentes causas, tais como a infecção pelo vírus 
Influenza, Parainfluenza, Adenovírus, Vírus Sincicial respiratório ou infecção por bactérias. A SG é definida como uma infecção respiratória caracterizada pela presença de febre de início súbito, mesmo que referida, acompanhada de tosse ou dor, além de outros sintomas como cefaleia, mialgia ou artralgia (Mazon, Komuchena, Roik, Wieczorkievicz, \& Ditterich, 2016).

Sobretudo entre os vírus responsáveis pelas SG, o vírus Influenza destaca-se como o mais prevalente e conhecido por causar surtos epidêmicos. Esse vírus é capaz de gerar uma infecção viral aguda do sistema respiratório de elevada transmissibilidade e de distribuição global, em que um mesmo indivíduo pode contraí-la várias vezes ao longo da vida e que, em geral, tem evolução autolimitada, apesar de também poder apresentar-se em forma grave (Taubenberger \& Morens, 2008).

$\mathrm{O}$ vírus influenza é subdividido nos tipos $\mathrm{A}, \mathrm{B}$ e $\mathrm{C}$, os quais podem sofrer mutações. $\mathrm{O}$ vírus influenza $\mathrm{C}$ causa apenas infecções respiratórias brandas, não possui impacto na saúde pública e não está relacionado com epidemias. Por outro lado, os vírus influenza A e B são responsáveis por epidemias sazonais, sendo o vírus influenza A responsável pelas grandes pandemias. Os vírus influenza A são ainda classificados em subtipos de acordo com as proteínas de superfície, sendo os subtipos mais comuns circulantes em humanos o $\mathrm{A}(\mathrm{H} 1 \mathrm{~N} 1)$ e $\mathrm{A}(\mathrm{H} 3 \mathrm{~N} 2)$. Alguns vírus influenza $\mathrm{A}$ de origem animal também podem infectar humanos causando doença grave, como os vírus $\mathrm{A}(\mathrm{H} 5 \mathrm{~N} 1), \mathrm{A}(\mathrm{H} 7 \mathrm{~N} 9), \mathrm{A}(\mathrm{H} 10 \mathrm{~N} 8)$, $\mathrm{A}(\mathrm{H} 3 \mathrm{~N} 2 \mathrm{v}), \mathrm{A}(\mathrm{H} 1 \mathrm{~N} 2 \mathrm{v})$, entre outros (Neumann \& Kawaoka, 2015).

Uma complexa relação entre infecções virais e vitamina $D$ tem sido apontada, que pode compreender a ativação do modo antiviral, a função imunomoduladora, a relação com elementos celulares e virais, a indução do processo de autofagia e morte celular programada, além de modificações genéticas e epigenéticas (Teymoori-Rad, Shokri, Salimi, \& Marashi, 2019). Rondanelli et al. (2018) refere em uma revisão que a vitamina $D$ pode atuar na redução do risco de resfriado comum por meio de mecanismos divididos em três categorias: barreira física, imunidade celular natural e adaptação a imunidade. Em condições clínicas, Vitamina D tem um importante função no tratamento primário ou adjuvante para infecções virais, bacterianas e fungais (Schwalfenberg, 2010).

A vitamina D aumenta a imunidade celular inata parcialmente através da indução de antimicrobianos peptídeos, incluindo catelicidina humana, LL-37, por 1,25-dihidroxivitamina D e defensinas (Grant et al., 2020a). As catelicidinas exibem atividades antimicrobianas diretas contra um espectro de micróbios, incluindo Bactérias Gram-positivas e Gramnegativas, vírus envelopados e não-envelopados e fungos. Esses peptídeos derivados do 
hospedeiro matam os patógenos invasores perturbando suas membranas celulares e podem neutralizar as atividades biológicas das endotoxinas (Agier, Efenberger, \& BrzezińskaBłaszczyk, 2015). Em um modelo de camundongo, o LL-37 reduziu a replicação do vírus influenza A (Barlow et al., 2011). Em outro estudo laboratorial, a 1,25 (OH) 2D reduziu a replicação do rotavírus in vitro e in vivo por outro processo (Zhao et al., 2019). Um ensaio clínico relatou que a suplementação com $4.000 \mathrm{UI} / \mathrm{d}$ de vitamina D diminuiu infecção pelo vírus dengue (Martínez-Moreno, Hernandez, \& Urcuqui-Inchima, 2019).

A vitamina $\mathrm{D}$ também aumenta a imunidade celular em parte reduzindo a tempestade de citocinas induzida pelo sistema imunológico inato. O sistema imunológico inato gera próinflamatórios e citocinas anti-inflamatórias em resposta a infecções virais e bacterianas, como observado em pacientes com COVID-19 (Huang et al., 2020). A vitamina D pode reduzir a produção de citocinas Th1 pró-inflamatórias como fator de necrose tumoral $\alpha$ e interferon $\gamma$. A administração de vitamina $\mathrm{D}$ reduz a expressão citocinas pró-inflamatórias e aumenta a expressão de citocinas anti-inflamatórias por macrófagos (Sharifi, Vahedi, Nedjat, Rafiei, \& Hosseinzadeh-Attar, 2019).

Em um estudo realizado por Gruber-Bzura (2018) sobre o papel da vitamina D na gripe foi observado que a vitamina $\mathrm{D}$ deve reduzir o risco de influenza, mas que são necessários mais estudos para avaliar essa possibilidade. Grandes estudos populacionais também seriam úteis, além do que a suplementação de vitamina D também está relacionada a alterações na concentração sérica de $25(\mathrm{OH}) \mathrm{D}$, que pode sofrer interferências de acordo com a região geográfica de cada país, por exemplo, são mais baixas na maioria dos países de latitude média e alta e, dessa forma, populações distintas no que concernem as concentrações de $25(\mathrm{OH}) \mathrm{D}$ (Grant et al., 2020b).

\subsection{Vitamina D e COVID-19}

Em relação à eficácia da vitamina D como uma possível forma de prevenção, ela pode atuar por meio de vários mecanismos pode reduzir o risco de infecções, por isso tem despertado o interesse da comunidade científica em investigar seu possível efeito na prevenção ou tratamento da COVID-19 (Grant et al., 2020b). Acredita-se que a vitamina D possa suprimir a proteína 2 complexadora de adenosina desaminase (CD26), uma molécula de adesão putativa para a invasão de células hospedeiras da COVID-19. Além disso, a vitamina $\mathrm{D}$ pode atenuar as respostas inflamatórias do interferon gama (IFN $\gamma$ ) e da 
interleucina-6 (IL-6), que são citocinas que aumentam em pacientes ventilados em estado crítico, incluindo aqueles com COVID-19 (McCartney \& Byrne, 2020).

Outro possível mecanismo de atuação da vitamina D é melhorar a expressão de genes relacionados à atividade antioxidante (subunidade modificadora da glutationa redutase e modificador da glutamato-cisteína ligase) (Lei, Zhang, Cheng, \& Lee, 2017). Com o aumento da produção de glutationa é poupado o uso de ácido ascórbico (vitamina C), que possui atividades antimicrobianas e que talvez possa ajudar a prevenir e tratar a COVID-19 (Colunga Biancatelli, Berrill, \& Marik, 2019; Mousavi, Bereswill, \& Heimesaat, 2019; Wimalawansa, 2020).

Aspecto que tem chamado a atenção na pandemia de COVID-19 é a maior morbimortalidade pela doença em idosos e em pessoas com comorbidades, como obesidade, hipertensão arterial, diabetes mellitus, entre outras. Vale destacar que as concentrações séricas de 25-hidroxivitamina D $25(\mathrm{OH}) \mathrm{D}$ tendem a diminuir com a idade, o que pode ajudar a explicar o aumento das taxas de letalidade pela COVID-19 com o aumento da idade (Vásárhelyi, Sátori, Olajos, Szabó, \& Bekő, 2011). Entre as possíveis explicações para as menores concentrações séricas dessa vitamina em idosos podem ser apontadas o menor tempo de exposição ao sol e a produção reduzida de vitamina $\mathrm{D}$ como resultado de níveis mais baixos de 7-desidrocolesterol na pele (MacLaughlin \& Holick, 1985). Além disso, alguns medicamentos (antiepiléticos, antineoplásicos, antibióticos, anti-inflamatórios, antihipertensivos, anti-retrovirais) reduzem as concentrações séricas de $25(\mathrm{OH}) \mathrm{D}$ e, geralmente, o uso desses fármacos, inclusive em politerapia, aumenta com a idade (Gröber \& Kisters, 2012).

Um estudo multicêntrico retrospectivo realizado na Ásia com 212 casos de COVID19 verificou que o nível sérico médio vitamina $\mathrm{D}$ foi de $23,8 \mathrm{ng} / \mathrm{ml}$, sendo os níveis mais baixos observados nos casos mais críticos de COVID-19 e níveis mais altos nos casos leves. Esses resultados podem indicar que um aumento nas concentrações séricas de vitamina D pode ajudar a melhorar as condições clínicas ou mitigar os piores resultados (graves a críticos), enquanto uma diminuição dos níveis sérios dessa vitamina pode estar relacionada com quadros clínicos mais graves em pacientes com COVID-19 (Alipio, 2020).

Existem orientações das autoridades de saúde da Irlanda e de todo o mundo de que pessoas mais velhas devam receber suplementação com vitamina $\mathrm{D}$; e existem evidências que demonstram segurança da suplementação de vitamina D3 em doses de 20 a $50 \mu$ g/dia. Sugerese que essa suplementação seja principalmente de todos os pacientes hospitalizados, residentes em casas de repouso e moradores da comunidade idosos com vitamina $\mathrm{D}$ em uma 
dose diária mínima de $20 \mu \mathrm{g} /$ dia (McCartney \& Byrne, 2020). Essa suplementação pode ser direcionada a outros constituintes vulneráveis (por exemplo, aqueles com diabetes mellitus ou função imunológica comprometida, aqueles com pele mais escura, vegetarianos e veganos, com sobrepeso ou obesidade, fumantes e profissionais de saúde) e, finalmente, estendidos ao restante da população, a fim de mitigar os graves riscos à saúde pública associados à infecção pelo COVID-19 (McCartney \& Byrne, 2020). Apesar das possíveis vias sugeridas para ação da vitamina D na COVID-19, os ensaios clínicos sobre sua utilização nos casos da doença ainda estão em andamento (Zhang, Xie, \& Hashimoto, 2020).

\section{Considerações Finais}

Considerando as ações da vitamina D no sistema imune inato e no aumento da capacidade quimiotática e fagocítica, essa vitamina tem se tornado tópico de discussão quanto à sua eficácia no combate a diversas doenças infectocontagiosas e, particularmente, como recurso auxiliar no manejo da COVID-19. A análise dos estudos incluídos nesta revisão evidencia que a vitamina $\mathrm{D}$ pode exercer funções imunomoduladoras e anti-inflamatórias com benefícios em infecções virais. Entretanto, apesar das possíveis vias de ação da vitamina D na modulação da resposta imune que poderiam contribuir na prevenção e tratamento da COVID19, recomenda-se o desenvolvimento de ensaios clínicos para melhor elucidar sua ação prática.

\section{Referências}

Agier, J., Efenberger, M., \& Brzezińska-Błaszczyk, E. (2015). Review paper cathelicidin impact on inflammatory cells. Central European Journal of Immunology, 2, 225-235. https://doi.org/10.5114/ceji.2015.51359

Alipio, M. (2020). Vitamin D supplementation could possibly improve clinical outcomes of patients infected with coronavirus-2019 (COVID-2019). SSRN Electronic Journal, 1-9. https://doi.org/10.2139/ssrn.3571484

Barlow, P. G., Svoboda, P., Mackellar, A., Nash, A. A., York, I. A., Pohl, J., ... Donis, R. O. (2011). Antiviral activity and increased host defense against influenza infection elicited by the 
Research, Society and Development, v. 9, n. 7, e771974614, 2020

(CC BY 4.0) | ISSN 2525-3409 | DOI: http://dx.doi.org/10.33448/rsd-v9i7.4614

$\begin{array}{lllll}\text { human } & \text { cathelicidin } & \text { LL-37. } & \text { PLOS }\end{array}$

https://doi.org/10.1371/journal.pone.0025333

Bikle, D. D., Malmstroem, S., \& Schwartz, J. (2017). Current controversies: are free vitamin metabolite levels a more accurate assessment of Vitamin D status than total levels? Endocrinology and Metabolism Clinics of North America, 46(4), 901-918. https://doi.org/10.1016/j.ecl.2017.07.013

Bikle, D. D., Patzek, S., \& Wang, Y. (2018). Physiologic and pathophysiologic roles of extra renal CYP27b1: Case report and review. Bone Reports, 8, 255-267. https://doi.org/10.1016/j.bonr.2018.02.004

Borella, E., Nesher, G., Israeli, E., \& Shoenfeld, Y. (2014). Vitamin D: a new anti-infective agent? Annals of the New York Academy of Sciences, 1317(1), 76-83. https://doi.org/doi: $10.1111 /$ nyas. 12321

Carlberg, C. (2017). Molecular endocrinology of vitamin D on the epigenome level. Molecular and Cellular Endocrinology, 453, 14-21. https://doi.org/10.1016/j.mce.2017.03.016

Castranova, V., Asgharian, B., Sayre, P., Virginia, W., \& Carolina, N. (2014). Metabolic Regulation of Immune Responses Kirthana. Annu Rev Immunol, 32(1), 609-634. https://doi.org/10.1080/10937404.2015.1051611.INHALATION

Chen, S., Sims, G. P., Chen, X. X., Gu, Y. Y., Chen, S., \& Lipsky, P. E. (2007). Modulatory Effects of 1,25-Dihydroxyvitamin D3 on Human B Cell Differentiation. The Journal of Immunology, 179(3), 1634-1647. https://doi.org/10.4049/jimmunol.179.3.1634

Chiang, C. M., Ismaeel, A., Griffis, R. B., \& Weems, S. (2016). Effects of vitamin D supplementation on muscle strength in athletes: A systematic review. Journal of Strength and Conditioning Research, 31(2), 566-574. 
Christakos, S., Dhawan, P., Verstuyf, A., Verlinden, L., \& Carmeliet, G. (2016). Vitamin D: Metabolism, Molecular Mechanism of Action, and Pleiotropic Effects. Physiological Reviews, 96(1), 365-408. https://doi.org/10.1152/physrev.00014.2015

Colunga Biancatelli, R. M. L., Berrill, M., \& Marik, P. E. (2019). The antiviral properties of vitamin C. Expert Review of Anti-Infective Therapy, 18(2), 99-101. https://doi.org/10.1080/14787210.2020.1706483

Czaja, A. J., \& Montano-Loza, A. J. (2018). Evolving role of Vitamin D in immune-mediated disease and its implications in autoimmune hepatitis. Digestive Diseases and Sciences, 64(2), 324-344. https://doi.org/10.1007/s10620-018-5351-6

Fisher, S. A., Rahimzadeh, M., Brierley, C., Gration, B., Doree, C., Kimber, C. E., ... Roberts, D. J. (2019). The role of vitamin D in increasing circulating $\mathrm{T}$ regulatory cell numbers and modulating $\mathrm{T}$ regulatory cell phenotypes in patients with inflammatory disease or in healthy volunteers: A systematic review. PLOS ONE, 14(9), e0222313. https://doi.org/10.1371/journal.pone.0222313

Gombart, A. F., Pierre, A., \& Maggini, S. (2020). A review of micronutrients and the immune system-working in harmony to reduce the risk of infection. Nutrients, 12(1), 236. https://doi.org/10.3390/nu12010236

Grant, W. B., Lahore, H., McDonnell, S. L., Baggerly, C. A., French, C. B., Aliano, J. L., \& Bhattoa, H. P. (2020a). Evidence that Vitamin D supplementation could reduce risk of influenza and COVID-19 infections and deaths. Nutrients, 12(4), E988. https://doi.org/10.20944/preprints202003.0235.v2

Grant, W. B., Lahore, H., McDonnell, S. L., Baggerly, C. A., French, C. B., Aliano, J. L., \& Bhattoa, H. P. (2020b). Vitamin D supplementation could prevent and treat influenza, $\begin{array}{llll}\text { coronavirus, and } & \text { pneumonia infections. }\end{array}$ https://doi.org/10.20944/preprints202003.0235.v1

Gröber, U., \& Kisters, K. (2012). Influence of drugs on vitamin D and calcium metabolism. Dermato-Endocrinology, 4(2), 158-166. https://doi.org/10.4161/derm.20731 
Gruber-Bzura, B. M. (2018). Vitamin D and influenza - prevention or therapy? $\begin{array}{llllll}\text { International Journal of } & \text { Molecular }\end{array}$ https://doi.org/10.3390/ijms19082419

Guillot, X., Semerano, L., Saidenberg-Kermanac'h, N., Falgarone, G., \& Boissier, M.-C. (2010). Vitamin D and inflammation. Joint Bone Spine, 77(6), 552-557. https://doi.org/10.1016/j.jbspin.2010.09.018

Haroon, M., \& FitzGerald, O. (2011). Vitamin D and its emerging role in immunopathology. Clinical Rheumatology, 31(2), 199-202. https://doi.org/10.1007/s10067-011-1880-5

Haussler, M. R., Jurutka, P. W., Mizwicki, M., \& Norman, A. W. (2011). Vitamin D receptor (VDR) - mediated actions of $1 \alpha, 25(\mathrm{OH})$ 2vitamin D3: Genomic and non-genomic mechanisms. Best Practice \& Research Clinical Endocrinology \& Metabolism, 25(4), 543559. https://doi.org/10.1016/j.beem.2011.05.010

Haussler, M. R., Whitfield, G. K., Kaneko, I., Haussler, C. A., Hsieh, D., Hsieh, J.-C., \& Jurutka, P. W. (2012). Molecular mechanisms of vitamin D action. Calcified Tissue International, 92(2), 77-98. https://doi.org/10.1007/s00223-012-9619-0

Holick, M. F. (1995). Vitamin D: Photobiology, metabolism, and clinical applications. In De Groot LC, ed. Endocrinology (7th ed., pp. 990-1011). Philadelphia: Elsevier.

Hornsby, E., Pfeffer, P. E., Laranjo, N., Cruikshank, W., Tuzova, M., Litonjua, A. A., ... Hawrylowicz, C. (2018). Vitamin D supplementation during pregnancy: Effect on the neonatal immune system in a randomized controlled trial. Journal of Allergy and Clinical Immunology, 141(1), 269-278.e1. https://doi.org/10.1016/j.jaci.2017.02.039

Huang, C., Wang, Y., Li, X., Ren, L., Zhao, J., Hu, Y., ... Cao, B. (2020). Clinical features of patients infected with 2019 novel coronavirus in Wuhan, China. The Lancet, 395(10223), 497-506. https://doi.org/10.1016/s0140-6736(20)30183-5 
Katona, P., \& Katona-Apte, J. (2008). The interaction between nutrition and infection. Clinical Infectious Diseases, 46(10), 1582-1588. https://doi.org/10.1086/587658

Kimball, A., Hatfield, K. M., Arons, M., James, A., Taylor, J., Spicer, K., ... Jernigan, J. A. (2020). Asymptomatic and presymptomatic SARS-CoV-2 infections in residents of a longterm care skilled nursing facility - King County, Washington, March 2020. MMWR. Morbidity and Mortality Weekly Report, 69(13), 377-381. https://doi.org/10.15585/mmwr.mm6913e1

Lei, G. S., Zhang, C., Cheng, B.-H., \& Lee, C.-H. (2017). Mechanisms of action of vitamin D as supplemental therapy for pneumocystis pneumonia. Antimicrobial Agents and Chemotherapy, 61(10). https://doi.org/10.1128/aac.01226-17

Lin, R. (2016). Crosstalk between Vitamin D metabolism, VDR signalling, and innate immunity. BioMed Research International, 2016, 1-5. https://doi.org/10.1155/2016/1375858

MacLaughlin, J., \& Holick, M. F. (1985). Aging decreases the capacity of human skin to produce vitamin D3. Journal of Clinical Investigation, 76(4), 1536-1538. https://doi.org/10.1172/jci112134

Martínez-Moreno, J., Hernandez, J. C., \& Urcuqui-Inchima, S. (2019). Effect of high doses of vitamin D supplementation on dengue virus replication, Toll-like receptor expression, and cytokine profiles on dendritic cells. Molecular and Cellular Biochemistry, 464(1-2), 169180. https://doi.org/10.1007/s11010-019-03658-w

Mazon, L. M., Komuchena, K. S., Roik, A. K., Wieczorkievicz, A. M., \& Ditterich, R. G. (2016). Perfil epidemiológico de pacientes com síndrome gripal e síndrome respiratória aguda grave. Saúde Em Revista, 16(43), 37-44. https://doi.org/10.15600/2238-1244/sr.v16n43p3744

McCartney, D. M., \& Byrne, D. G. (2020). Optimisation of vitamin D status for enhanced immuno-protection against COVID-19. Ir Med J., 113(4), 58. 
McKenna, N. J., Cooney, A. J., DeMayo, F. J., Downes, M., Glass, C. K., Lanz, R. B., ... O'Malley, B. W. (2009). Minireview: evolution of NURSA, the nuclear receptor signaling atlas. Molecular Endocrinology, 23(6), 740-746. https://doi.org/10.1210/me.2009-0135

Mousavi, S., Bereswill, S., \& Heimesaat, M. M. (2019). Immunomodulatory and antimicrobial effects of vitamin C. European Journal of Microbiology and Immunology, 9(3), 73-79. https://doi.org/10.1556/1886.2019.00016

Neumann, G., \& Kawaoka, Y. (2015). Transmission of Infleunza A viruses. Virology, (480), 234-246. https://doi.org/10.1016/j.virol.2015.03.009.Transmission

Oda, Y., Sihlbom, C., Chalkley, R. J., Huang, L., Rachez, C., Chang, C.-P. B., ... Bikle, D. D. (2003). Two distinct coactivators, DRIP/mediator and SRC/p160, are differentially involved in Vitamin D receptor transactivation during keratinocyte differentiation. Molecular Endocrinology, 17(11), 2329-2339. https://doi.org/10.1210/me.2003-0063

Oliveira, A., Sofia Vilela, S., Warkentin, S., Araújo, J., Ramos, E., \& Lopes, C. (2020). Da emergência de um novo vírus humano à disseminação global de uma nova doença. ISPUP.

Ou, X., Liu, Y., Lei, X., Li, P., Mi, D., Ren, L., ... Qian, Z. (2020). Characterization of spike glycoprotein of SARS-CoV-2 on virus entry and its immune cross-reactivity with SARSCoV. Nature Communications, 11(1), 1620. https://doi.org/10.1038/s41467-020-15562-9

Prosser, D. E., \& Jones, G. (2004). Enzymes involved in the activation and inactivation of vitamin D. Trends in Biochemical Sciences, 29(12), 664-673. https://doi.org/10.1016/j.tibs.2004.10.005

Rokni, M., Ghasemi, V., \& Tavakoli, Z. (2020). Immune responses and pathogenesis of SARS-CoV-2 during an outbreak in Iran: Comparison with SARS and MERS. Reviews in Medical Virology, 1-6. https://doi.org/10.1002/rmv.2107

Rondanelli, M., Miccono, A., Lamburghini, S., Avanzato, I., Riva, A., Allegrini, P., ... Perna, S. (2018). Self-care for common colds: the pivotal role of Vitamin D, Vitamin C, zinc, and echinacea in three main immune interactive clusters (physical barriers, innate and adaptive 
immunity) involved during an episode of common colds — practical advice on dosages . Evidence-Based Complementary and Alternative Medicine, 2018, 1-36. https://doi.org/10.1155/2018/5813095

Sakaki, T., Sawada, N., Komai, K., Shiozawa, S., Yamada, S., Yamamoto, K., ... Inouye, K. (2000). Dual metabolic pathway of 25-hydroxyvitamin D3 catalyzed by human CYP24. European Journal of Biochemistry, 267(20), 6158-6165. https://doi.org/10.1046/j.14321327.2000.01680.x

Schwalfenberg, G. K. (2010). A review of the critical role of vitamin D in the functioning of the immune system and the clinical implications of vitamin D deficiency. Molecular Nutrition \& Food Research, 55(1), 96-108. https://doi.org/10.1002/mnfr.201000174

Schwartz, J. B., Gallagher, J. C., \& Jorde, R. (2018). Determination of free 25 (OH) D concentrations and their relationships to total $25(\mathrm{OH})$ D in multiple clinical populations. $J$ Clin Endocrinol Metab, 103(9), 3278-3288.

Scully, C., Georgakopoulou, E. A., \& Hassona, Y. (2017). The immune system: Basis of so much health and disease: 4. immunocytes. Dental Update, 44(5), 436-442. https://doi.org/10.12968/denu.2017.44.5.436

Sharifi, A., Vahedi, H., Nedjat, S., Rafiei, H., \& Hosseinzadeh-Attar, M. J. (2019). Effect of single-dose injection of vitamin $\mathrm{D}$ on immune cytokines in ulcerative colitis patients: a randomized placebo-controlled trial. APMIS, 127(10), 681-687. https://doi.org/10.1111/apm.12982

Sociedade Brasileira de Pediatria. (2014). Deficiência de vitamina D em crianças a adolescentes. Sociedade Brasileira de Pediatria, 99(1), 1132-1141.

Taubenberger, J. K., \& Morens, D. M. (2008). The pathology of influenza virus infections. Annu Rev Pathol, 3(1), 499-522. 
Teymoori-Rad, M., Shokri, F., Salimi, V., \& Marashi, S. M. (2019). The interplay between vitamin D and viral infections. Reviews in Medical Virology, 29(2), e2032. https://doi.org/10.1002/rmv.2032

Trochoutsou, A. I., Kloukina, V., Samitas, K., \& Xanthou, G. (2015). Vitamin-D in the immune system: genomic and non-genomic actions. Mini-Reviews in Medicinal Chemistry, 15(11). https://doi.org/10.2174/1389557515666150519110830

Vásárhelyi, B., Sátori, A., Olajos, F., Szabó, A., \& Bekő, G. (2011). Low vitamin D levels among patients at Semmelweis University: retrospective analysis during a one-year period. Orvosi Hetilap, 152(32), 1272-1277. https://doi.org/10.1556/oh.2011.29187

Whitfield, G. K. (1996). Vitamin D receptors from patients with resistance to 1,25dihydroxyvitamin D3: point mutations confer reduced transactivation in response to ligand and impaired interaction with the retinoid $\mathrm{X}$ receptor heterodimeric partner. Molecular Endocrinology, 10(12), 1617-1631. https://doi.org/10.1210/me.10.12.1617

Wimalawansa, S. J. (2020). Global epidemic of coronavirus-COVID-19: What we can do to minimze risks. Eur J Biomed Pharm Sci, 7, 432-438.

Wintergerst, E. S., Maggini, S., \& Hornig, D. H. (2007). Contribution of selected vitamins and trace elements to immune function. Annals of Nutrition and Metabolism, 51(4), 301-323. https://doi.org/10.1159/000107673

Wu, D., Lewis, E. D., Pae, M., \& Meydani, S. N. (2019). Nutritional modulation of immune function: analysis of evidence, mechanisms, and clinical relevance. Frontiers in Immunology, 9. https://doi.org/10.3389/fimmu.2018.03160

Yan, C. H., Faraji, F., Prajapati, D. P., Boone, C. E., \& DeConde, A. S. (2020). Association of chemosensory dysfunction and Covid-19 in patients presenting with influenza-like symptoms. International Forum of Allergy \& Rhinology, 1-18. https://doi.org/10.1002/alr.22579 
Zhang, J., Xie, B., \& Hashimoto, K. (2020). Current status of potential therapeutic candidates for the COVID-19 crisis. Brain, Behavior, and Immunity, S0889-1591(20)30589-4. https://doi.org/10.1016/j.bbi.2020.04.046

Zhao, Y., Ran, Z., Jiang, Q., Hu, N., Yu, B., Zhu, L., \& Chen, D. (2019). Vitamin D alleviates rotavirus infection through a Microrna-155-5p mediated regulation of the TBK1/IRF3 signaling pathway in vivo and in vitro. International Journal of Molecular Sciences, 20(14), 3562. https://doi.org/10.3390/ijms20143562

\title{
Porcentagem de contribuição de cada autor no manuscrito
}

\author{
Valmir Oliveira Silvino - 12,5\% \\ Mayara Maria Lima Pereira - 12,5\% \\ Rayane Carvalho de Moura - 12,5\% \\ Mara Cristina Carvalho Batista - 12,5\% \\ Bruno Viana Rosa $-12,5 \%$ \\ Eduardo Henrique de Moura - 12,5\% \\ Maria do Carmo Carvalho e Martins - 12,5\% \\ Marcos Antonio Pereira dos Santos - 12,5\%
}

